RBMC

ISSN 2447-9071

doi https://doi.org/10.36414/rbmc.v7i19.109

Contato para correspondência: Ana Paula Felix Arantes

E-mail:

ana_paula_arantes@hotmail.com

Conflito de interesse: Não

Financiamento: Recursos próprios

Recebido: 31/10/2021

Aprovado: 07/12/2021

\section{Saúde bucal de crianças com trissomia do cromossomo 21}

\section{Oral health in children with chromosomal trisomy 21}

Heloysa Melo de Freitas ${ }^{1}$, Renato Canevari Dutra da Silva ${ }^{1}$, Ana Paula Felix Arantes ${ }^{1}$, Marcos Marcondes de Godoy ${ }^{1}$

${ }^{1}$ Universidade de Rio Verde - UniRV

\section{Resumo}

A trissomia do cromossomo 21, também conhecida popularmente como Síndrome de Down, éuma alteração genética que gera alterações físicas e congênitas, através de uma modificação no cromossomo número 21, a qual pode ocasionar diversas alterações orais e físicas no paciente. Devido a estas alterações, os portadores desta trissomia necessitam de tratamento e avaliação odontológicos especializados além de diagnóstico precoce. Este estudo possuiu como objetivo realizar, por meio de revisão de literatura narrativa, uma pesquisa sobre o atendimento odontológico a portadores da trissomia do cromossomo 21 e suas principais alterações bucais. Os resultados demonstraram que os portadores da síndrome possuem diversas alterações relacionadas ao sistema estomatognático, tais como anodontias, respiração bucal, língua fissurada, atraso e modificação da sequência de erupção dos dentes, geminação, incisivos centrais em meia lua, fusão de dentes, comprometimento da articulação temporomandibular. O referencial consultado revelou que o atendimento precoce, desdeo primeiro mês de vida, com abordagem preventiva pode refletir em melhora das condições de saúde bucal deste grupo de pacientes.

Palavras-Chave: Saúde bucal. Odontopediatria. Trissomia do cromossomo 21.

\begin{abstract}
Chromosome 21 trisomy, also popularly known as Down syndrome, is a genetic alteration that causes physical and congenital alterations, through a modification in chromosome number 21, which can cause several oral and physical alterations in the patient. Due to these changes, patients with this trisomy need specialized dental treatment and evaluation, in addition to early diagnosis. This study aimed to carry out, through a review of narrative literature, a research on dental care for patients with trisomy 21 and its main oral alterations. The results showed that patients with the syndrome have several changes related to the stomatognathic system, such as anodontia, mouth breathing, fissured tongue, delay, and modification of the teeth eruption sequence, gemination, crescent-shaped central incisors, teeth fusion, impairment of the teeth. ear-jaw articulation. The reference consulted revealed that early care, from the first month of life, with a preventive approach, may reflect an improvement in the oral health conditions of this group of patients.
\end{abstract}

Keywords: Oral health. Pediatric dentistry. Trisomy 21. 


\section{Introdução}

A trissomia do cromossomo 21 é considerada uma alteração genética que altera a vida do portador no seu desenvolvimento, ocasionando algumas alterações físicas e cognitivas. Essa anomalia está relacionada a modificação no número de cromossomos do par 21, em razão de um erro na separação em uma das células dos pais, causando a presença de um cromossomo extra ${ }^{1}$.

Os portadores da trissomia do cromossomo 21 apresentam características tanto físicas, quanto orais, como exemplo: baixa estatura, anodontias, respiração bucal, língua fissurada, atraso e modificação da sequência de erupção dos dentes, geminação, incisivos centrais em meia lua, fusão de dentes, comprometimento da articulação temporomandibular ${ }^{2}$.

As doenças bucais e malformações orofaciais são considerados de teor para acarretar risco de morte, e com isso, os pacientes mais propensos a esses tipos de problemas devem receber uma atenção especial para diagnóstico e tratamento precoce $^{3}$.

A taxa de atendimento a pacientes especiais em consultórios odontológicos atualmente é baixo, em razão da falta de informação e comprometimento dos cirurgiões dentistas, em decorrência da ausência de contato com estes pacientes durante sua formação. O conhecimento das lesões e problemas ocasionados nesses pacientes devido a síndrome, também é considerado insuficiente, ocasionando assim um déficit na saúde oral deste grupo de pacientes ${ }^{4}$.

Com base neste contexto, este trabalho tem como objetivo apresentar as alterações bucais provocadas pela trissomia do cromossomo 21 e analisar a atuação do cirurgião dentista frente aos cuidados que devem ser tomados nos portadores da trissomia.

\section{Métodos}

Esta revisão bibliográfica narrativa foi realizada a partir da síntese de artigos científicos sobre a temática da saúde bucal em pacientes portadores de Trissomia do Cromossomo 21. Primeiramente foi realizado o levantamento bibliográfico, o qual foi feito através da busca nas seguintes bases de dados: Scientific Eletronic Library On-Line (Scielo), Google Acadêmico e PubMed. Foram utilizados os descritores em saúde: "saúde bucal", "assistência odontológica"e "trissomia do cromossomo 21 ", combinados entre si pelo operador Booleano "AND".

Foram incluídos estudos clínicos que relataram tratamentos odontológicos em pacientes com trissomia do cromossomo 21 , bem como ensaios clínicos randomizados, relatos de caso e livros-texto envolvendo esse tema, publicados entre os anos 1990 a 2020. Foram excluídos artigos que não apresentaram relevância clínica sobre o tema abordado, estudos repetidos, trabalhos de conclusão de cursos, bem como dissertações de mestrado e teses de doutorado, além dos estudos com metodologia incompletas e resultados inconclusivos. Após a leitura crítica de títulos e resumos, foram selecionados 33 estudos (Figura 1).

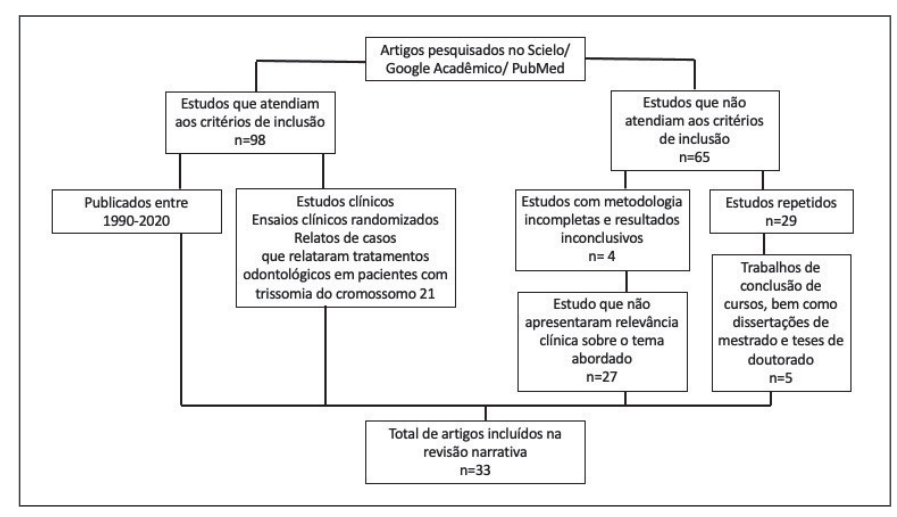

Figura 1. Fluxograma para a busca de artigos científicos.

\section{Resultados}

\section{Características da trissomia do cromossomo 21}

A trissomia do cromossomo 21 é uma alteração genética que possui influência na vida do portador devido a afetar seu desenvolvimento, determinando algumas características físicas e cognitivas. Também conhecida como Sindrome de Down, é considerada a anomalia genética mais comum. Essa anomalia ocorre com relação a alteração do número de cromossomos do par 21, em razão a um erro na separação destes em uma das células dos pais, criando a presença de um cromossomo extra'.

Mães em estágio avançado de idade possuem um risco elevado de terem filhos com trissomia do cromossomo 21, devido ao envelhecimento dos ovócitos, que pode provocar a destruição das fibras cromossômicas ou a deterioração do centrômero. Algumas pesquisas demonstram que a idade paterna também pode ser considerado um fator contribuinte ${ }^{5}$.

É realizado o diagnóstico da trissomia através da observação de sinais e sintomas e com a confirmação por um exame com estudo cromossômico (cariótipo), que consegue detectar um cromossomo 21 extra. Vale destacar que não se trata de uma doença, mas de uma condição inerente aquele indivíduo portador dessa anomalia, com isso, não se pode falar em cura ou tratamento, e sim no controle das condições sistêmicas e locais do indivíduo ${ }^{6}$.

Os portadores da trissomia normalmente apresentam um desenvolvimento facial diferente em relação a hipotonia mus- 
cular, que acomete inclusivamente o sistema estomatognático, a hiperflexibilidade de todo o corpo e apresentam um envelhecimento precoce. Os portadores da síndrome normalmente apresentam um volume cerebral menor, incluindo reduções no córtex parietal e no lobo temporal7.

Em relação aos aspectos cognitivos, a característica mais presente nos portadores da trissomia é a deficiência mental, que varia de indivíduo para indivíduo ${ }^{8}$ e as alterações neurológicas nesses indivíduos acabam acarretando em um déficit de atenção .

De forma clínica, pode-se notar uma dismorfia craniofacial e braquicefalia, fazendo com que a cabeça seja desproporcionalmente grande, o rosto se apresenta tipicamente hipotônico a língua fica visível e a boca permanece aberta, a face normalmente é achatada, o pescoço curto e achatado, e a estatura baixa, porém as mãos e pés pequenos e $\operatorname{largos}^{6,10}$.

Em relação as características presentes na cavidade bucal, os pacientes costumam apresentar: língua fissurada, anodontias, respiração bucal, micrognatia, atraso e modificação da sequência de erupção dentária, hipotonia com tendência a protuir a língua e permanecer de boca aberta, baixa prevalência de cárie, hipodontia, fissura nos cantos dos lábios, geminação, fusão de dentes, comprometimento da articulação temporomandibular, implantação irregular dos dentes e incisivos em meia lua'. Porém, nem todos os portadores desta síndrome possuem estes fenótipos, sendo a deficiência mental a única característica presente em todos os casos. Com o diagnostico e identificação do portador já no nascimento, aumenta os níveis de intervenção precoce auxilia todos os familiares envolvidos ${ }^{11}$.

O indivíduo portador da trissomia do cromossomo 21, além da possibilidade de apresentar as manifestações orais citadas, pode ainda ter um comprometimento do desenvolvimento da linguagem, apresentando-se mais lenta, e com isso, a criança acometida acaba possuindo atrasos no desenvolvimento neuropsicomotor ${ }^{12}$.

A incidência da síndrome é cerca de 1 (um) caso a cada 600 a 700 nascidos vivos, possuindo um maior índice nas mães com idade superior a 30 anos $^{13}$. Os pacientes possuem uma expectativa de vida entre 35 a 40 anos, sendo valido observar que esse indicador vem aumentando em decorrência da melhora da assistência à saúde ${ }^{14}$.

\section{Aspectos bucais e implicações odontológicas}

São diversas manifestações bucais presentes em pacientes da trissomia do cromossomo 21, incluindo: mandíbula e cavidade bucal pequenas, palato estreito, alto e ogival e língua que se apresenta frequentemente fissurada e grande, podendo manifestar glossite migratória benigna (língua geográfica) ${ }^{11}$.

A postura da língua aberta é comum em razão de uma nasofaringe estreita, bem como tonsilas e adenóide hiper trofiada. Devido a protusão da língua e a respiração bucal frequentes, ocorre a secura e fissura dos lábios. Podemos observar a presença de queilite angular nas comissuras labiais por causa da dificuldade do indivíduo em fechar a boca. Este movimento da língua pode acarretar também o deslocamento dos dentes e a má oclusão ${ }^{15}$.

O fato dos portadores da síndrome serem na maioria das vezes respiradores bucais, há um favorecimento no aparecimento de periodontite crônica e de infecções do trato respiratório. Como eles permanecem nesses casos com a boca aberta, babam frequentemente e o lábio inferior se apresenta gretado, podendo assim ocorrer fissuras nos lábios ${ }^{10}$.

Anomalias características e doenças periodontais são prevalentes nessa síndrome. Dentre as anomalias dentais que podem ser associadas a pacientes com trissomia do cromossomo 21 , as mais frequentes são: oligodontia, hipodontia, fusão, microdontia e taurodontia. Anomalias dentárias de desenvolvimento, como malformações coronárias e radiculares também se apresentam comuns. Variações morfométricas ligadas aos segundos molares decíduos são encontradas facilmente nos portadores da síndrome ${ }^{16}$.

Ocorrem também os casos de desarmonias oclusais, mordidas cruzadas posteriores, apinhamento pronunciado dos dentes e apertognatia nos portadores da trissomia do cromossomo 2117. A relação do alto índice de má oclusão pode ser relacionado com frequentes alterações na arcada superior, mais precisamente na região de incisivos e caninos, característica a qual ocorre pelo fato do arco maxilar apresentar-se pequeno e ogival, relacionado à macroglossia e outros aspectos etiológicos exógenos ${ }^{18}$.

Os portadores de trissomia do cromossomo 21 possuem muita das vezes um atraso na erupção dental, normalmente ocorrendo aos 6 meses e presente tanto na dentição decídua quanto na permanente e possuem um fluxo salivar em média $50 \%$ menor do que o normal, sendo associado ao metabolismo da glândula parótida. Além de que o pH salivar e os níveis de sódio, cálcio e bicarbonato serem mais altos12,15.

A prevalência de cárie dental se mostra não ser maior do que o normal nos portadores desta trissomia, porém ocorre uma apresentação de perda óssea elevada em relação a média, e com isso ocorre uma significativa propensão ao desenvolvimento de uma doença periodontal, aumentando as chances no decorrer do tempo ${ }^{18}$.

As principais características que predispõem o aparecimento da cárie nos portadores da trissomia é a presença de 
fissuras oclusais estreitas e profundas, ou sulcos vestibulares/ linguais, e a falta de uma higiene adequada também aumenta a incidência de cárie, podendo assim trazer problemas de infecções generalizadas se não tratado ${ }^{19}$.

Os fatores exógeneos associados ao aumento da progressão da doença periodontal nos pacientes portadores da trissomia do cromossomo 21 incluem a falta de higiene bucal, má oclusão, presença de matéria alba e cálculo, macroglossia e um hábito constante de manter a boca entreaberta ${ }^{8}$.

\section{Alterações bucais comuns em portadores da trissomia}

A trissomia do cromossomo 21 possui diversas manifestações bucais, incluindo: palato estreito, alto e ogival, mandíbula e cavidade bucal pequenas e a língua que se apresenta frequentemente fissurada e grande, podendo apresentar glossite migratória benigna (língua geográfica) ${ }^{11}$.

É normal que os portadores possuam a postura da língua aberta, graças a nasofaringe estreita, bem como tonsilas e adenóide hiper trofiada. A protrusão da língua e respiração bucal são frequentes devido a secura e fissura dos lábios. Na região das comissuras labiais, é visto a presença de queilite angular, em razão da dificuldade que o indivíduo possui em fechar a boca ${ }^{15}$.

Os dentes apresentam anomalias características e doença periodontal predominante. Dentre as anomalias dentais que são mais frequentes associadas a trissomia estão: microdontia, fusão, oligodontia, hipodontia e taurodontia. Sendo a mais frequente destas a microdontia. As anomalias dentárias de desenvolvimento, como as malfomarções coronárias e radiculares também são comuns. Variações morfométricas contendo os segundos molares decíduos são encontradas de forma frequente nos portadores da trissomia do cromossomo $21^{20,21}$.

Mordidas cruzadas posteriores, apertognatia, desarmonias oclusais e apinhamento pronunciado dos dentes são comuns nos portadores. O elevado índice da má oclusão está associado a alterações na arcada superior, sendo precisamente na região de caninos e incisivos. A característica se deve ao fato do arco maxilar se apresentar pequeno e ogival, associado à macroglossia e outros fatores etiológicos exógenos ${ }^{15,18}$.

$O$ atraso na erupção é frequentemente encontrado nos pacientes da trissomia, ocorrendo normalmente aos 6 meses e estando presente tanto na dentição decídua como na permanente e o fluxo salivar dos pacientes portadores da trissomia são em média 50\% menor do que em pessoas normais. Esta redução está associada preferencialmente ao metabolismo da glândula parótida. Além do mais, o pH salivar é mais alto, assim como os níveis de sódio, bicardonato e cálcio ${ }^{12}$.

A incidência e prevalência de cárie em portadores de trissomia é inferior quando comparada, porém os pacientes apresentam uma significativa propensão ao desenvolvimento de doença periodontal, aumentando de acordo com a idade ${ }^{19}$.

\section{Consequências das afecções odontológicas da crianças com T21}

Os pacientes com trissomia do cromossomo 21 devem possuir a participação dos seus pais e responsáveis constantemente dando estimulações nas atividades profiláticas e de manutenção da saúde bucal e naturalmente, os responsáveis dos portadores desta síndrome, passam grande parte do tempo cuidando de diversas tarefas e cuidados relacionados à síndrome, e com isso, acabam deixando de lado questões ligadas a prevenção de doenças bucais nesses indivíduos ${ }^{22}$.

O cuidador desses pacientes não recebe normalmente as instruções necessárias quanto aos cuidados direcionados aos dentes e à boca do portador da trissomia do cromossomo 21, fazendo assim que haja um aumento no risco dessa parcela de população desenvolver doenças bucais ${ }^{23}$.

Isso reflete na compreensão do cuidados sobre a importância da saúde bucal na qualidade de vida e na inclusão social dos portadores da síndrome e apesar de haver uma prevalência de doenças bucais, principalmente a doença periodontal e a cárie, sendo relacionada diretamente com a higiene oral, existe um grande percentual de pais e responsáveis que não identifica a escovação como uma tarefa de prioridade no indivíduo com trissomia do cromossomo $21^{24}$.

Uma problema causado diretamente devido a falta de higiene oral no indivíduo portador da síndrome é a halitose. Na literatura o mau hálito não é retratado em crianças com trissomia do cromossomo 21, porém pode se tornar presente nos adultos portadores da síndrome, devido a fatores que se tornam cada vez mais preentes na cavidade oral como a higiene oral precária, a respiração bucal, a motricidade oral deficiente, as maloclusões e os problemas periodontais, sendo que a língua dos portadores da síndrome é bastante fissurada, e essas fissuras acabam provocando um acúmulo de restos alimentares que propovocam halitose ${ }^{22,24}$.

Os problemas bucais envolvem não somente a saúde física do portador da trissomia do cromossomo 21, mas também o seu bem-estar econômico, social e psicológico, e são capazes até mesmo de influenciar na autoestima e consequente qualidade de vida destes indivíduos ${ }^{25}$.

\section{Prevenção das alterações bucais}

Nas doenças orais é importante para evitar o aparecimento ou progressão é necessário uma boa higiene e profilaxia. A fase de manutenção e controle de doença deve ser realizado de 3 em 3 meses, com inclusão de raspagens e alisamento radicular, 
uso de clorexidina e antibioticoterapia sistémica nos casos de doenças periodontais se necessário ${ }^{23}$.

O apoio familiar é extremamente fundamental na gestão de doenças bucais, e o uso do fio dental e das escovas mecanizadas para os pacientes portadores da trissomia do cromossomo 21 podem possuir grande utilidade, porém o uso do fio dental pode ser difícil para alguns pacientes ${ }^{27}$.

O nível de motivação do paciente interfere diretamente na manutenção da higiene oral. Se o paciente estiver motivado para realizar a higiene oral, o controle das doenças bucais será mais eficaz ${ }^{28}$. Na consulta dos pacientes portadores da síndrome, um membro da família deve estar sempre presente e deve receber juntamente com o paciente as explicações com natureza clara e objetiva para um entendimento satisfatório dos problemas que podem gerar a falta da higiene bucal e do acompanhamento ao cirurgião dentista. Devem também ser incluidas dietas saudáveis e procedimentos diários com a supervisão dos resposáveis com uso de flúor tópico e bochechos de flúor ${ }^{29}$.

A primeira consulta é essencial, pois além de um diagnóstico e de uma programação criteriosa do tratamento da criança portadora da síndrome, é avaliado o grau da ansiedade tanto dos pais quanto do paciente, e também deve ser avaliado o relacionamento familiar. É importante que seja apresentado as dependências do consultório aos pais e ao paciente, devido ao estímulos ambientes e emocionais poderem afetas no comportamento do paciente. Os pais ou os responsáveis pelo paciente devem ser aconselhados a não falar claramente sobre suas vivências e sobre seu medo em relação ao tratamento odontológico, devido a poder trazer ao paciente uma expectativa negativa e consequentemente um medo ${ }^{30}$.

O planejamento do atendimento ao paciente portador da síndrome deve ser dividido em quatro partes, sendo elas ${ }^{31}$ :

- Fase sistêmica: que abrange a investigação dos dados sobre a saúde geral do paciente, com a solicitação de exames complementares;

- Fase preparatória: possui o objetivo de adequar o meio bucal, fazendo a remoção de focos de infecção e recomendando uma mudança de hábitos do paciente e seus familiares;

- Fase restauradora: possui a função de restabelecer a forma e função dos dentes, possibilitando uma oclusão correta;

- Fase de manutenção: possui uma extrema importância, já que os pacientes especiais devem realizar visitas periódicas ao cirurgião dentista, em busca de preservar a saúde bucal.

Grande maioria dos pacientes que buscam um atendimento odontológico, já apresentam o diagnóstico de sua anormalidade firmado. É necessário que haja uma interação entre o cirurgião-dentista e os outros profissionais que atendem o paciente, principalmente o médico. Assim, o atendimento se torna multiprofissional ${ }^{30}$.

Os pacientes portadores da trissomia do cromossomo 21 normalmente são atendidos seguindo-se alguns critérios que facilitam a consulta, tais como: atendimentos realizados prefereencialmente em dupla (o cirurgião-dentista e um auxiliar treinado), com a presença do responsável pelo paciente, tempo da consulta não deve exceder 30 minutos $^{31}$.

Equipamentos de contensão física, caneta de alta rotação com a cabeça pequena, micromotor, sugador de saliva potente e abridores de boca de vários tamanhos são essenciais estar ao alcance do cirurgião dentista durante o atendimento para que seja agilizado além do uso de anestésicos nos procedimentos que provoquem uma sintomatologia dolorosa ${ }^{31}$.

O consultório deve ser equipado com todos os materiais necessários na emergência médica e deve possuir uma auxiliar que tenha conhecimento dos equipamentos, medicamentos e manobras do suporte básico de vida, e saiba o número do pronto socorro mais proxímo, devido à durante o momento dos atendimentos, os pacientes especiais possuem um risco maior de emergências ${ }^{32}$.

Para a prevenção dos problemas bucais nos pacientes portadores da trissomia do cromossomo 21 , foi desenvolvido a seguinte tabela com os cuidados a serem tomadas de acordo com a idade do paciente ${ }^{33}$ :

Quadro 1. Protocolo de cuidados rrelacionados à saúde bucal para a criança portadora da trissomia do cromossomo $21^{33}$

\begin{tabular}{|l|l|}
\hline \multicolumn{1}{|c|}{ IDADE } & \multicolumn{1}{c|}{ RECOMENDAÇõES } \\
\hline \multirow{3}{*}{ 0-3 anos } & $\begin{array}{l}\text {-Amamentação materna é o melhor suprimento alimentar para os } \\
\text { bebês. } \\
\text {-A criança deve tentar ingerir líquidos a partir dos seis meses de } \\
\text { idade através de um copo, evitando o uso da mamadeira. } \\
\text {-Não deve ser adicionado açucar aos alimentos. } \\
\text {-Os adultos precisam supervisionar ou escovar os dentes da criança. } \\
\text {-Usar uma pequena quantidade de pasta dental. }\end{array}$ \\
$\begin{array}{l}\text {-Assim que surgir o primeiro dente escovar antes de dormir e após } \\
\text { as refeições. }\end{array}$ \\
\hline 3-6 anos & $\begin{array}{l}\text {-Escovar os dentes da criança ao acordar, antes de dormir e após as } \\
\text { refeições, sempre com supervisão. } \\
\text {-A quantidade da pasta deve ser do tamanho de uma ervilha. } \\
\text {-O açucar não deve ser consumido mais de quatro vezes ao dia. } \\
\text {-Dê preferência a remédios que não contenham açucar. }\end{array}$ \\
\hline $\begin{array}{l}\text { A partir dos } \\
7 \text { anos }\end{array}$ & $\begin{array}{l}\text {-Escovar os dentes ao acordar, antes de dormir e após as refeições, } \\
\text { sempre com supervisão. } \\
\text {-Usar pasta de dente com flúor. } \\
\text {-O açucar deve ser consumido menos de quatro vezes ao dia, e evi- } \\
\text { tar alimentos e medicamentos que contenham açucar. } \\
\text {-Fazer o uso de enxaguante bucal com flúor todos os dias em um } \\
\text { horário diferente ao da escovação, sempre com supervisão. }\end{array}$ \\
\hline
\end{tabular}




\section{Atuação do odontólogo frente as arbitrariedades odontológicas da criança com T21}

Um indivíduo que possui um déficit intelectual leve pode ser atendimento pelo cirurgião dentista no consultório odontológico, e a anestesia geral é indicada nos casos que as técnicas de condicionamento não funcionam, ou quanto existem uma deficiência mental grave?

Nos procedimentos cirúrgicos, é necessário ser feito uma antibioterapia profilática, com o parecer do médico cardiologista do paciente, graças às alterações cardíacas congênitas presentes em grande parte dos portadores ${ }^{28}$.

Os cuidados em relação a higiene bucal desses pacientes são redobrados, em razão do alto risco de doença periodontal precoce e normalmente o padrão da higiene oral desses pacientes é muito pobre, devido ao retardo físico e metal, sendo necessário que o profissional redobre esse tipo de atenção durante os atendimentos e procedimentos. Os auxiliares para profilaxia envolvem clorexidina, escovas dentais automáticas, enxaguatórios, dieta não cariogênica e pastas fluoretadas, além de raspagens regulares ${ }^{30}$.

É necessário que haja o incentivo para as mães dos portadores da trissomia aumentarem a prática do aleitamento materno, em razão da hipotonicidade muscular generalizada. A fonoaudiologia tem contribuição para promoção de um estímulo precoce na musculatura ${ }^{31}$.

O paciente portador da trissomia do cromossomo 21 possui tanto manifestações sistêmicas quanto orais, sendo essencial o atendimento que inclua o conceito de globalidade e que disponibilize aos pacientes serem assistidos por uma equipe multidisciplinar ${ }^{3}$.

Normalmente esses pacientes devem ir com mais frequência no consultório dentário. Os profissionais normalmente encontram alguns dificuldades em realizar o atendimento, porém o consultório deve reunir determinadas condições adequadas e ergonômicas de acessibilidade, como por exemplo portas largas, corrimãos para apoio, rampas para cadeiras de rodas ${ }^{7}$.

Normalmente os cirurgiões dentistas se sente pouco à vontade diante do atendimento de pacientes especiais, há uma falta de sensibilidade e confiança, e a remuneração é desadequada ${ }^{22}$. O profissional deve focar na sua abordagem no nível de compreensão e da comunicação com a criança. A comunicação entre o cirurgião dentista e o paciente deve ser com contacto ocular e a atuação do odontopediatra deve ser direcionada à criança com a finalidade de conseguir apurar quais as suas motivações para criar estratégias que deixem a criança interessada e que colabore no tratamento ${ }^{27}$.

Sendo assim os problemas dentários ligados a trissomia do cromossomo 21 podem ser reduzidos ou até mesmo elimi- nados quando acompanhados por médicos dentistas desde a fase da dentição decídua ${ }^{3}$.

\section{Conclusão}

Foram analisados vários estudos para observação das alterações bucais dos portadores da trissomia do cromossomo 21, diante dos quais foi concluído que referida alteração genética promove diversas alterações bucais, que se não consideradas durante a abordagem odontológica, podem levar a problemas sérios de saúde, e que se diagnosticadas precocemente o tratamento pode ser realizado com sucesso.

As doenças na cavidade oral do paciente portador da trissomia do cromossomo 21 podem acarretar problemas de saúde, comprometimento na rotina diária do indivíduo, alterações comportamentais e dificuldades de interação social, o que gera a necessidade de um atendimento de acordo com suas necessidades, de caráter integral, com dimensão dos fatores de risco à saúde e com enfoque preventivo.

A integralidade já declarada não se define com a reprodução de protocolos ou rotinas, o prossional deve ter conhecimento e compreensão do contexto de vida do paciente para ser capaz de adaptar a sua atuação no contexto de cada consulta. São necessários mais estudos sobre a temática da trissomia do cromossomo 21 acerca das alterações bucais e seu manejo, buscando enfoque preventivo na abordagem terapêutica, utilizando-se de diagnóstico precoce e preconizando a qualidade de vida destes pacientes.

\section{Referências}

1. Oliveira RMB de, Junior PA de A. Sensibilização para o Cuidado em Saúde Bucal em Pacientes com Síndrome de Down. Ciência Atual - Revista Científica Multidisciplinar do Centro Universitário São José.2017;10(2).

2. Acerbi AG, de Freitas $C$, de Magalhães MH. Prevalence of numeric anomalies in the permanent dentition of patients with Down syndrome. Spec Care Dentist. 2001;21(2):75-8.

3. Oliveira AC, Czeresnia D, Paiva SM, Campos MR, Ferreira EF. Uso de serviços odontológicos por pacientes com síndrome de Down. Rev Saúde Pública. 2008;42(4):693-9.

4. Falcão AC de SLA, Santos JM dos, Nascimento KLL, Santos DB do N, Costa PV de A. Síndrome de Down: abordagem odontopediátrica na fase oral. Revista de Odontologia da Universidade Cidade de São Paulo. 2019;31(1):57-67.

5. Kwon JY, Park IY, Kwon S-M, Kim CJ, Shin JC. The quadruple test for Down syndrome screening in pregnant 
women of advanced maternal age. Arch Gynecol Obstet. 2012;285(3):629-33.

6. Carvalho ACA de, Campos PSF, Crusoé-Rebello I. Síndrome de Down: aspectos relacionados ao sistema estomatognático. CMBIO. 2010;9(1):49.

7. Silva ZCM da, Pagnoncelli SD, Weber JBB, Fritscher AMG. Avaliação do perfil dos pacientes com necessidades especiais da clínica de odontopediatria da Faculdade de Odontologia da PUCRS. Rev odonto ciênc. 2005;20(50):313-8.

8. Pereira Macho VM, Seabra M, Pinto A, Soares D, De Andrade $C$. Alterações craniofaciais e particularidades orais na trissomia 21. Portuguese Journal of Pediatrics. 2014; 190-194.

9. Wuo AS. A construção social da Síndrome de Down. Cadernos de Psicopedagogia. 2007;6(11):00-00.

10. Santangelo CN, Gomes DP, Vilela L de O, Deus TS de, Vilela V de O, Santos EM. Avaliação das características bucais em pacientes portadores de síndrome de Down da APAE de Mogi das Cruzes - SP. Cons Saúde.2008;7(1):29-34.

11. Reimand T, Uibo O, Zordania R, Palmiste V, Ounap $\mathrm{K}$, Tqlvik T. Parents' satisfaction with medical and social assistance provided to children with Down syndrome: experience in Estonia. Community Genet. 2003;6(3):166-70.

12. Schwartzman JS. Sindrome de Down. Sao Paulo: Memnon; 2003.

13. Gabriela Talita C, Mascarello AP, Bardini DR, Fracaro GB, Boleta-Ceranto D de CF. O papel do cirurgião-dentista na manutenção da saúde bucal de portadores de síndrome de down. Odontologia Clínico-Científica (Online). 2011;10(3):247-50.

14. Carvalho ACA de, Campos PSF, Crusoé-Rebello I. Síndrome de Down: aspectos relacionados ao sistema estomatognático. cmbio. 2010;9(1):49.

15. Regezi JA, Moleri AB, David FP, Moreira LC, Tavares RR. Patologia bucal: correlações clinico patológicas. Rio de Janeiro: Guanabara Koogan; 2000.

16. Peretz B, Shapira J, Farbstein H, Arieli E, Smith P. Modification of tooth size and shape in Down's syndrome. J Anat. 1996;188(Pt 1):167-72.

17. Mestrović SR, Rajić Z, Papić JS. Hypodontia in patients with Down's syndrome. Coll Antropol. 1998;22 Suppl:69-72.

18. Ondarza A, Jara L, Bertonati MI, Blanco R. Tooth malalignments in Chilean children with Down syndrome. Cleft Palate Craniofac J. 1995;32(3):188-93.
19. Gabre P, Martinsson T, Gahnberg L. Longitudinal study of dental caries, tooth mortality and interproximal bone loss in adults with intellectual disability. Eur J Oral Sci. 2001;109(1):20-6.

20. Townsend GC. Tooth size in children and young adults with trisomy 21 (Down) syndrome. Arch Oral Biol. 1983;28(2):159-66.

21. Peretz B, Katzenel V, Shapira J. Morphometric variables of the primary second molar in children with Down syndrome. J Clin Pediatr Dent. 1999;23(4):333-6.

22. Hennequin M, Allison PJ, Veyrune JL. Prevalence of oral health problems in a group of individuals with Down syndrome in France. Dev Med Child Neurol. 2000;42(10):691-8.

23. Oliveira AC, Luz CLF, Paiva SM. O papel da saúde bucal na qualidade de vida do indivíduo com síndrome de Down. Arq Odontol.2007;43(4).

24. Marcondes E, Vaz FAC, Ramos JLA, Okay Y. Pediatria básica: pediatria geral e neonatal. 9. ed. São Paulo: Sarvier; 2002.

25. De Oliveira ACB, De Paiva SM, Pordeus IA. Fatores relacionados ao uso de diferentes métodos de contenção em pacientes portadores de necessidades especiais. BDS. 2010;7(3).

26. Raggio DP, Takeuti ML, Guaré R de O, Haddad AS, Imparato JCP, Ciamponi AL. Remoção químico-mecânica de tecido cariado em paciente portador de síndrome de Down: relato de caso clínico. JBP, j bras odontopediatr odontol bebê. 2001;191-6.

27. Pilcher E. Dental care for the patient with down syndrome. Down Syndrome Research and Practice. 1998;5(3):111-6.

28. Elias R. Odontologia de Alto Risco - Pacientes especiais. Rio de Janeiro: Revinter; 1995.

29. Rodríguez Guerrero K, Clavería Clark RA, Peña Sisto M. Algunas características clinicoepidemiológicas del síndrome de Down y su repercusión en la cavidad bucal. MEDISAN. 2015;19(10):1272-82.

30. Mugayar FRL. Pacientes portadores de necessidades especiais: Manual de Odontologia e Saúde Oral. São Paulo: Pancast; 2000.

31. Correa MSNP. Odontopediatria na primeira infância. 2. ed. São Paulo: Editora Santos; 2005.

32. Haddad AS. Odontologia para pacientes com necessidades especiais. São Paulo: Editora Santos; 2007.

33. Bull MJ, Committee on Genetics. Health supervision for children with Down syndrome. Pediatrics. 2011;128(2):393-406. 